Homology, Homotopy and Applications, vol.12(1), 2010, pp.11-26

\title{
UNSTABLE MODULE PRESENTATIONS FOR THE COHOMOLOGY OF REAL PROJECTIVE SPACES
}

\author{
DAVID J. PENGELLEY AND FRANK WILLIAMS
}

(communicated by Donald M. Davis)

\begin{abstract}
There is much we still do not know about projective spaces. We describe here how the mod two cohomology of each real projective space is built as an unstable module over the Steenrod algebra $\mathcal{A}$, or equivalently, over $\mathcal{K}$, the algebra of inherently unstable mod two "lower operations" originally introduced by Steenrod. In particular, to produce the cohomology of projective space of each dimension we consider the well-known minimal set of unstable module generators and construct a minimal set of unstable relations. Three new perspectives we blend for this purpose are:

- to focus solely on the two-power Steenrod squares that generate $\mathcal{A}$ to understand the $\mathcal{A}$-action in a process we call "shoveling ones",

- to describe every element in a canonical way from a particular unstable generator by composing operations from the algebra $\mathcal{K}$,

- to shift attention when studying an unstable $\mathcal{A}$-module to considering and analyzing it directly as an equivalent $\mathcal{K}$-module.
\end{abstract}

\section{Introduction}

Much of the structure of the real projective spaces $\mathbb{R} P(m)$ and their limit $\mathbb{R} P(\infty)$ is captured in their mod two cohomologies, which are unstable algebras over the Steenrod algebra $\mathcal{A}$. As algebras their structure could hardly be simpler: $H^{*} \mathbb{R} P(\infty) \cong \mathbb{F}_{2}[t]$ with $\operatorname{deg}(t)=1$, and the inclusions $\mathbb{R} P(m) \subset \mathbb{R} P(\infty)$ induce quotients $H^{*} \mathbb{R} P(m) \cong$ $\mathbb{F}_{2}[t] /\left(t^{m+1}\right)$. As unstable algebras over $\mathcal{A}$, their structure is uniquely determined by the basic properties of unstable $\mathcal{A}$-algebras, namely the unstable dimension condition, the Cartan formula, and the topological consequence that $S q^{0}$ acts as the identity [8].

Received July 14, 2009, revised October 27, 2009; published on February 18, 2010.

2000 Mathematics Subject Classification: 55R35, 55S05, 55S10.

Key words and phrases: Steenrod algebra, unstable module, Kudo-Araki-May algebra, real projective space, presentation.

This article is available at http://intlpress.com/HHA/v12/n1/a2

Copyright (C) 2010, International Press. Permission to copy for private use granted. 
From these it is obvious that the action is given by

$$
S q^{k}\left(t^{n}\right)=\left(\begin{array}{l}
n \\
k
\end{array}\right) t^{n+k},
$$

where $\mathcal{A}$ is generated as an algebra by the Steenrod squares $\left\{S q^{k} \mid k \geqslant 0\right\}$, which are subject to the Adem relations [8]. However, the structure of these cohomology algebras as unstable $\mathcal{A}$-modules is nonetheless very elaborate. For instance, we can ask what are minimal sets of $\mathcal{A}$-generators and $\mathcal{A}$-relations, an important question for applications.

We will describe such minimal unstable presentations for the cohomology of all these projective spaces in the category of unstable $\mathcal{A}$-modules. First we describe minimal $\mathcal{A}$-presentations for $H^{*} \mathbb{R} P(\infty)$ and its natural $\mathcal{A}$-filtration pieces $F_{s} H^{*} \mathbb{R} P(\infty)$. Our $\mathcal{A}$-presentation for $H^{*} \mathbb{R} P(\infty)$ is in [6, Theorem 6.5], but the proofs there are circuitous and rely on methods that are ill-suited to our primary goal here of minimally presenting the cohomologies $H^{*} \mathbb{R} P(m)$ of the finite projective spaces from the presentations of $\left.F_{s} H^{*} \mathbb{R} P(\infty)\right)$. We thus propose a new perspective, taking three distinctive and atypical points of view on unstable modules, and we provide proofs of all our results from this perspective.

As an initial indication of our results, we state now a form of our minimal presentation of $H^{*} \mathbb{R} P(\infty)$, which will emerge from the detailed theorems that follow.

Recall first that there is a filtration $F_{s}=F_{s} H^{*} \mathbb{R} P(\infty)$, for $s=0,1, \ldots, \infty$, of $\mathcal{A}$-submodules given by the $\mathbb{F}_{2}$-subspace $F_{s}=\mathbb{F}_{2}\left\{t^{r} \mid \alpha(r) \leqslant s\right\}$, where $\alpha(r)$ is the number of ones in the binary expansion of the nonnegative integer $r$. That these vector subspaces are $\mathcal{A}$-closed is clear from the action formula above.

Minimal presentation for $F_{s} H^{*} R P(\infty)$ (see Theorem 3.1 and remark below). For $0 \leqslant s \leqslant \infty$, a minimal presentation of $F_{s} H^{*} \mathbb{R} P(\infty)$ as an unstable module is given by generators

$$
x_{2^{j}-1}, \text { for } j \text { an integer with } 0 \leqslant j \leqslant s \quad \text { (degree of } x_{2^{j}-1} \text { is } 2^{j}-1 \text { ), }
$$

and relations

$$
S q^{2^{k}} x_{2^{j}-1}=S q^{2^{j-1}} S q^{2^{k}} x_{2^{j-1}-1}, \text { for } 0 \leqslant j \leqslant s \text { and } 0 \leqslant k \leqslant j-2 .
$$

To prove this and our minimal presentations for the finite projective spaces, we develop the following three points of view.

1. Shoveling ones. Our first point of view is to study the $\mathcal{A}$-action entirely in terms of the set of two-power Steenrod squares $\left\{S q^{2^{i}} \mid i \geqslant 0\right\}$ that minimally generates $\mathcal{A}$. We do this partly because the cohomology of a projective space has at most one nonzero element in each degree, and the two-power squares connect these in a manner particularly easily viewed in terms of the binary expansions of their degrees; so all calculations will reduce to simple relationships between binary expansions. Additionally, we shall see that the minimal set of $\mathcal{A}$-relations forming a projective space is easily described using just these two-power squares, in a process we call 'shoveling ones', especially when combined with our second point of view.

2. Represent with $\mathcal{K}$. Our second point of view appears at first sight to contradict the first. It is to label and conceive of all elements in unstable modules primarily via iteration of Steenrod's original unstable "cup- $i$ " squares on the $\mathcal{A}$-generators of our 
module. We denote these operations by $\left\{D_{j} \mid j \geqslant 0\right\}$. They generate the Kudo-ArakiMay algebra $\mathcal{K}$, with its own set of $\mathcal{K}$-Adem relations [5]. The $D_{j}$ are related to Steenrod squares via Steenrod's defining relationship $S q^{i} x_{m}=D_{m-i} x_{m}$ on a class $x_{m}$ of degree $m$. The homology analogue to our point of view is already standard in studying the homology of loop spaces $[\mathbf{1}, \mathbf{2}, \mathbf{3}, \mathbf{4}]$. While the $S q^{i}$ are stable operations in that they are preserved by suspension, the $D_{j}$ are not. We find that the form of monomials using the unstable $D_{j}$ is often much more useful than those in the stable $S q^{i}$ for describing elements and bases in unstable modules. For projective spaces the $D_{j}$ are particularly efficacious since we can relate their iterations well to the binary expansions of degrees of elements. This allows us to choose a very useful canonical representation for each element in terms of a particular monomial in the $D_{j}$ on a particular unstable module generator, which in turn enables us to analyze the global module structure. To wit, we will prove (cf. [6, Theorems 6.1, 6.2])

A basis for $H^{*} R P(\infty)$ in terms of $\mathcal{K}$ (from Theorem 2.1). For $s \geqslant 0$, a basis for $F_{s} H^{*} \mathbb{R} P(\infty) / F_{s-1} H^{*} \mathbb{R} P(\infty)$ in terms of admissibles from $\mathcal{K}$ on $t^{2^{s}-1}$ is given by

$$
\left\{D_{0}^{e_{0}} \cdots D_{2^{i}-1}^{e_{i}} \cdots D_{2^{s-1}-1}^{e_{s-1}} t^{2^{s}-1} \mid \text { each } e_{i} \geqslant 0\right\}
$$

with corresponding degrees $\sum_{i=0}^{s-1} 2^{e_{0}+\cdots+e_{i}+i}$ having $\alpha$-number $s$. (Notice that the $e_{i}$ are simply the lengths of the blocks of consecutive zeros in the binary expansions of degrees.)

The first two viewpoints are not contradictory: we use the $D_{j}$ to represent elements for a basis, and the $S q^{2^{i}}$ to see how the module action connects them, with both viewpoints closely tied together via binary expansions of degrees. Of course to meld the two points of view effectively we must be comfortable working flexibly and simultaneously with both the $S q^{2}$ and the $D_{j}$ in whatever combination is most judicious.

3. Study unstable $\mathcal{A}$-modules as $\mathcal{K}$-modules. This brings us to our third viewpoint, which goes beyond the second, to view our unstable modules interchangeably over the Steenrod algebra $\mathcal{A}$ or the Kudo-Araki-May algebra $\mathcal{K}$. In particular, the proof of the minimal presentation theorem above for $F_{s} H^{*} \mathbb{R} P(\infty)$ relies on shifting to module analyses entirely over $\mathcal{K}$.

We end the introduction with a statement of our main result on presentations for finite projective spaces. To state it, we first note how to recast the $\mathcal{A}$-relations of the presentation of $F_{s} H^{*} \mathbb{R} P(\infty)$ in terms of the points of view just espoused.

Remark. By induction on $j$ one sees that the set of relations

$$
S q^{2^{k}} x_{2^{j}-1}=S q^{2^{j-1}} S q^{2^{k}} x_{2^{j-1}-1}, \quad \text { for } 0 \leqslant j \leqslant s \text { and } 0 \leqslant k \leqslant j-2,
$$

is equivalent to the set

$$
S q^{2^{k}} x_{2^{j}-1}=S q^{2^{j-1}} S q^{2^{j-2}} \cdots S q^{2^{k}} x_{2^{k+1}-1}, \quad \text { for } 0 \leqslant j \leqslant s \text { and } 0 \leqslant k \leqslant j-2 \text {, }
$$

and the latter is equal to the set

$$
S q^{2^{k}} x_{2^{j}-1}=D_{2^{k}-1}^{j-k} x_{2^{k+1}-1}, \quad \text { for } 0 \leqslant j \leqslant s \text { and } 0 \leqslant k \leqslant j-2 .
$$


Our main theorem of this paper is the following.

Minimal presentation for finite projective spaces (Theorem 4.2). Let $2^{s}-1 \leqslant$ $m \leqslant 2^{s+1}-2$. Let $\alpha$ be the number of ones in the binary expansion of $m+1$, and write the expansion as $m+1=\sum_{i=0}^{\alpha-1} 2^{e_{0}+\cdots+e_{i}+i}$ (each $e_{i} \geqslant 0$ ). Also let $\beta$ be the number of leading ones in this expansion; in other words, if there is a nonzero $e_{i}$, then $\alpha-\beta$ is the largest index $i$ for which $e_{i}$ is nonzero.

Then a minimal presentation for $H^{*} \mathbb{R} P(m)$ is given by generators

$$
x_{2^{j}-1} \text { in degree } 2^{j}-1, \text { for } 0 \leqslant j \leqslant s,
$$

and the three sets of relations

$$
\begin{gathered}
S q^{2^{k}} x_{2^{j}-1}=D_{2^{k}-1}^{j-k} x_{2^{k+1}-1}, \text { for } 0 \leqslant j \leqslant s \text { and } 0 \leqslant k \leqslant j-2, \\
0=D_{2^{i+r}-1}^{e_{i}-l} D_{2^{i+1+r}-1}^{e_{i+1}} \cdots D_{2^{\alpha-1+r}-1}^{e_{\alpha-1}} x_{2^{\alpha+r}-1}, \\
\text { for } 0 \leqslant i \leqslant \alpha-1 \text { and } 0 \leqslant l<e_{i}, \text { where } r=e_{0}+\cdots+e_{i-1}+l, \text { and } \\
0=D_{2^{k}-1}^{s+1-k} x_{2^{k+1}-1}, \text { for } s+1-\beta \leqslant k \leqslant s-1 .
\end{gathered}
$$

Remark. While the first relations are simply those we used to present $F_{s} H^{*} \mathbb{R} P(\infty)$ above, it is not easy to get a sense for the second and third set of relations from this formulaic description; they are best understood in terms of visualizing binary expansions. In Section 4 where we prove the theorem, we first illustrate the theorem with the special family where $m$ is of the form $2^{s+1}-2$ (in which case the second set of relations is empty). Notice that the third set of relations for $H^{*} \mathbb{R} P\left(2^{s+1}-2\right)$ consists of the additional relations that would appear if we instead presented $F_{s+1} H^{*} \mathbb{R} P(\infty)$ as above, and then set the unnecessary generator $x_{2^{s+1}-1}$ on the left side equal to zero. This is why we write relations (b) and (c) with zero on the left.

Example. For $m=6$, of form $2^{s+1}-2$ with $s=2$, we have that $H^{*} \mathbb{R} P(6)$ is minimally presented as an unstable $\mathcal{A}$-module by generators $x_{1}$ and $x_{3}$ with the relation $S q^{1} x_{3}=D_{0}^{2} x_{1}=S q^{2} S q^{1} x_{1}$ of the first kind, and relations $0=D_{0}^{3} x_{1}=S q^{4} S q^{2} S q^{1} x_{1}$ and $0=D_{1}^{2} x_{3}=S q^{4} S q^{2} x_{3}$ of the third kind.

Remark. In Section 4 we also discuss in the general case all the relations from the point of view of binary expansions, the interplay between the second and third sets of relations, and give another concrete example, for a more general $m$, in terms of binary expansions. When thought of in terms of binary expansions, it is not difficult to visualize how the main theorem follows from the minimal presentation theorem above for $F_{s} H^{*} \mathbb{R} P(\infty)$.

\section{Acknowledgements}

We thank the referee for very helpful suggestions on exposition. 


\section{Two points of view}

\subsection{Two-power squares and shoveling ones}

To flesh out our first point of view, given $n \geqslant 0$, with binary expansion $n=$ $\sum_{i=0} a_{i} 2^{i}$ (i.e., $a_{i}=0$ or 1 ), we will often write $\left(a_{0} \cdots a_{i} \cdots\right)$ for $n$; in other words, we may denote $n$ simply by listing the digits of its binary expansion (enclosed in parentheses), but in the reverse order from what is ordinary when writing numbers in decimal form. The reader will soon see why reverse listing of the binary digits is helpful to our work. Sometimes we need explicitly to indicate the exponent of the power of two corresponding to a digit (also called its 'place' or 'position'), so we may write

$$
\left(a_{0} \cdots a_{i} \cdots\right)
$$

to indicate this. Note that an ellipsis may stand for any combination of digits. We will write $\left(\cdots a_{l} \leftrightarrow a_{k} \cdots\right)$ to mean that $a_{l}=a_{j}=a_{k}$ for all $l \leqslant j \leqslant k$. Although the nonzero elements of the cohomology of $H^{*} \mathbb{R} P(\infty) \cong \mathbb{F}_{2}[t]$ are in one-to-one correspondence with the binary expansions of their degrees, we wish to have explicit corresponding labels for the elements themselves, so in degree $n=\left(a_{0} \cdots a_{i} \cdots\right)$ we write $t\left(a_{0} \cdots a_{i} \cdots\right)$ for the element $t^{n}$.

Now we can observe how two-power squares act in relation to binary expansions. From equation (1), the action of $S q^{2^{i}}$ on an arbitrary element $x=t\left(a_{0} \cdots a_{i} \cdots\right)$ is clearly zero if $a_{i}=0$, while if $a_{i}=1$, the result is nonzero and can be written as follows, since it is the unique nonzero element in degree

$$
\left(a_{0} \cdots a_{i} \cdots\right)+(0 \leftrightarrow 0 \underset{i}{ }) .
$$

Write

$$
x=t\left(\cdots{ }_{i}^{11} \leftrightarrow \underset{j}{10} \cdots\right)
$$

i.e., $j$ is the first place above $i$ with a zero digit. Then since

$$
(\cdots \underset{i}{11} \leftrightarrow \underset{j}{10} \cdots)+(0 \leftrightarrow 0 \underset{i}{01})=(\cdots \underset{i}{00} \leftrightarrow \underset{j}{01} \cdots),
$$

we have

$$
S q^{2^{i}} x=S q^{2^{i}} t\left(\cdots{ }_{i}^{11} \leftrightarrow \underset{j}{10} \cdots\right)=t(\cdots \underset{i}{00} \leftrightarrow \underset{j}{01} \cdots) .
$$

Notice in sum, then, that the effect of applying $S q^{2}$ to the nonzero element in a degree with digit 1 in place $i$ is to replace that digit 1, along with any consecutively above it, with zeros, and to convert the first zero encountered above place $i$ into a 1. We refer to this procedure on binary expansions as 'shoveling ones', since we imagine pushing the 1 in place $i$ rightwards into the first open (i.e., value 0 ) place $j$, and sweeping away any consecutive intervening ones. We will call a shovel 'simple' if there are no intervening ones (i.e., $j=i+1$ ), and 'longer' if $j>i+1$, so that some ones are actually swept into oblivion by our shovel.

From this shoveling perspective we immediately notice several things (some already well-known) intimately relating the $\mathcal{A}$-action on projective spaces with $\alpha$-number of degrees. Indeed, since two-power squares generate $\mathcal{A}$, and since shoveling obviously 
never increases $\alpha$-number, the $\mathcal{A}$-action respects the filtration $F_{s} H^{*} \mathbb{R} P(\infty)$ by $\alpha$ number defined in the introduction. Notice too that a simple shovel leaves $\alpha$-number unchanged, while a longer shovel reduces it. Moreover, from this point of view it is obvious that

$$
\left\{t^{2^{j}-1}=t(1 \leftrightarrow \underset{j-1}{1}) \mid j \geqslant 0\right\}
$$

is the unique minimal set of $\mathcal{A}$-generators of $H^{*} \mathbb{R} P(\infty)$, since clearly none of these elements is in the image of a shovel (which always produces a 0 to the left of a 1), and any binary expansion is the image of simple shovels from the generator with the same $\alpha$-number, for instance by 'simply' shoveling ones rightward as needed into their desired places, beginning at the right. And it is equally clear that these same elements for $j \leqslant s$ are the minimal set of generators of the filtration $F_{s}$. We shall call the elements of this minimal set 'minimal generators'.

\subsection{Candidates for a minimal set of $\mathcal{A}$-relations}

In seeking necessary $\mathcal{A}$-relations in a minimal presentation for $F_{s} H^{*} \mathbb{R} P(\infty)$, we should clearly first consider, for a fixed minimal generator $t^{2^{j}-1}$ with $j \leqslant s$, which generators of lower filtration it may be related to and how. Since two-power squares generate $\mathcal{A}$, the first possibilities to consider are $S q^{2^{k}}\left(t^{2^{j}-1}\right)$ for $0 \leqslant k \leqslant j-1$. We have, from above, the shovel

$$
S q^{2^{k}}\left(t^{2^{j}-1}\right)=S q^{2^{k}} t(1 \leftrightarrow \underset{j-1}{1})=t(1 \leftrightarrow \underset{k}{10} \underset{j}{01}) \text { if } k \leqslant j-1
$$

While for $k=j-1$ this equation involves a simple shovel, and yields no connection to lower filtration, for $0 \leqslant k \leqslant j-2$ it involves longer shovels, and we have thus discovered essential and independent connections between filtrations, so that relations corresponding to these, at a minimum, must be included in any presentation of $F_{s} H^{*} \mathbb{R} P(\infty)$. We shall show that precisely this family of relations minimally suffices to present $F_{s} H^{*} \mathbb{R} P(\infty)$.

First we need to decide how each element on the right side in the above equalities should best be represented via $\mathcal{A}$-action on a minimal generator, in order to write down a claimed abstract presentation. For this we need to flesh out our second point of view, since there may be a multitude of ways to represent such an element via module action on minimal generators.

\subsection{Representing basis elements by iterating unstable operations $D_{j}$ from $\mathcal{K}$}

We will do more than just represent the particular elements of our candidate relations above. We will represent every element of $H^{*} \mathbb{R} P(\infty)$ as a preferred chosen monomial in the $D_{j}$ 's applied to a preferred minimal $\mathcal{A}$-generator.

We remind the reader that the unstable operations $D_{j}$ compose very differently from the Steenrod squares, and form their own algebra of operations $\mathcal{K}$, the KudoAraki-May algebra. Despite this algebra being extremely different from $\mathcal{A}$, unstable modules over $\mathcal{K}$ are in perfect correspondence with those over $\mathcal{A}$. Let us recall [5] just a few basic features of $\mathcal{K}$, and how it will inform what we mean by an 'unstable module'. 
The bigraded algebra $\mathcal{K}$ is generated by elements $D_{i} \in \mathcal{K}_{1, i}, i=0,1, \ldots$, subject to the (Adem) relations

$$
D_{i} D_{j}=\sum_{k}\left(\begin{array}{c}
k-1-j \\
2 k-i-j
\end{array}\right) D_{i+2 j-2 k} D_{k}, \quad(i>j) .
$$

The degree of elements in $\mathcal{K}$ satisfies the condition that multiplication be a map $\mathcal{K}_{m, i} \otimes \mathcal{K}_{n, j} \rightarrow \mathcal{K}_{m+n, i+2^{m} j}$. The identity element is $1 \in \mathcal{K}_{0,0}$. A monomial $D_{I}=$ $D_{i_{1}} \cdots D_{i_{n}}$ is admissible provided the multi-index $I=\left(i_{1}, \ldots, i_{n}\right)$ is nondecreasing, and the admissibles form a basis for $\mathcal{K}$. A graded $\mathcal{K}$-module $M_{*}$ is one that satisfies the requirement $\mathcal{K}_{m, i} \otimes M_{j} \rightarrow M_{2^{m} j-i}$. It is unstable provided that each $D_{i}: M_{i} \rightarrow M_{i}$ is the identity and that $D_{i}\left(M_{k}\right)=0$ for $i>k$. Unstable modules over $\mathcal{K}$ and $\mathcal{A}$ always correspond, via the relationship $S q^{i} x_{m}=D_{m-i} x_{m}$ on a class of degree $m$, so in the sequel we shall refer simply to an unstable module without preference for either $\mathcal{A}$ or $\mathcal{K}$. Note too that $D_{0} x_{m}=S q^{m} x_{m}$. In particular, this should permanently dispel any misapprehension that might confuse $D_{0} \in \mathcal{K}_{1,0}$ with the identity element $1 \in \mathcal{K}_{0,0}$. For the cohomology of a space, the structure and correspondence extends further to that of an unstable $\mathcal{K}$-algebra, with its own Cartan formula, but we will not need that here.

To advance our second point of view, we now assign our preferred representation to each element in $H^{*} \mathbb{R} P(\infty)$ (and thence in its filtrations $F_{j}$ and quotients $H^{*} \mathbb{R} P(m)$ ) as a single admissible monomial in $\mathcal{K}$ applied to a preferred minimal module generator. It is clear from our shoveling discussion above that an arbitrary element may have a myriad of representations via iterated operations on various possible minimal module generators. We intend to single one out first by matching the $\alpha$-number $j$ of the degree of our arbitrarily chosen element $x$ with the minimal generator of the same $\alpha$-number, shunning all representations that reduce $\alpha$. Even with the same $\alpha$-number, there will generally be numerous ways to choose and to order iteration of operations that will carry our chosen generator $t^{2^{j}-1}=t(1 \leftrightarrow 1)$ to the given $x$. Since the binary expansions of these two degrees both have $j$ ones, they differ by the placement of the $j$ blocks of zeros (some possibly empty) in the expansion of the degree of $x$, one to the left of each of its $j$ ones.

We will use these blocks to dictate a specific admissible in the $D$ 's to produce $x$ from $t^{2^{j}-1}$. First note that in $H^{*} \mathbb{R} P(\infty)$ the criterion for whether $D_{i}\left(t^{n}\right)$ is nonzero is given by $\left(\begin{array}{c}n \\ i\end{array}\right)$, since

$$
D_{i}\left(t^{n}\right)=S q^{n-i}\left(t^{n}\right)=\left(\begin{array}{c}
n \\
n-i
\end{array}\right) t^{2 n-i}=\left(\begin{array}{c}
n \\
i
\end{array}\right) t^{2 n-i} .
$$

Our plan is to express $x$ from $t^{2^{j}-1}$ not necessarily by iterating simple shovels (via two-power squares), but more efficiently, by inserting a new needed zero in the degree expansion with each operation, in a way that pushes the entire part of the degree expansion to the right of the insertion point rightwards one position to accommodate the new zero. And we insert the new zeros working from right to left. For the first block of zeros this amounts to simple shoveling via two-power squares, since only a single one is being shoveled, but after this block non-shovels will be used, since multiple ones separated by zeros are being pushed rightwards. Nonetheless, we shall now see that when all is expressed in terms of $D$ 's, the process is quite transparent. 
The key is to observe how a single operation can insert a zero after (i.e., to the right of in the reversed binary expansion) trailing ones (we call ones "trailing" if they are at the left of the reversed binary expansion, representing the least significant digits), thus shifting an entire initial part of the expansion rightwards one position. We claim this will be accomplished by

$$
D_{2^{k}-1} t\left(1 \leftrightarrow{ }_{k-1}^{1} \cdots\right)=t\left(1 \leftrightarrow{ }_{k-1}^{1} 0 \cdots\right),
$$

where the two ellipses represent the same string (shifted right by the operation $\left.D_{2^{k}-1}\right)$. This holds because the resulting degree is clearly

$$
2 \cdot\left(1 \leftrightarrow{ }_{k-1}^{1} \cdots\right)-\left(2^{k}-1\right)=\left(1 \leftrightarrow{ }_{k-1}^{1} 0 \cdots\right),
$$

and the result is nonzero because

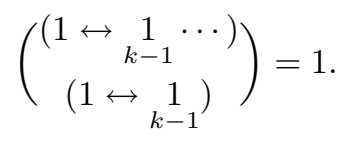

Thus it is clear that for an arbitrary degree

$$
(\underbrace{0 \leftrightarrow 0}_{e_{0}} 1 \cdots \underbrace{0 \leftrightarrow 0}_{e_{i}} 1 \cdots \underbrace{0 \leftrightarrow 0}_{e_{j-1}} 1)=\sum_{i=0}^{j-1} 2^{e_{0}+\cdots+e_{i}+i}
$$

with $\alpha$-number $j$ (note some of the $e_{i}$ may be zero), iterations of the above nature on $t^{2^{j}-1}$, working from right to left through the blocks, lead to a particular admissible monomial involving only $D$ 's of the form $D_{2^{k}-1}$, for $k<j$, applied to $t^{2^{j}-1}$, and that the degrees and their elements are in one to one correspondence with such monomials in $\mathcal{K}$. For example, for $j=8$,

$$
t(0011000110110011)=D_{0}^{2} D_{3}^{3} D_{15} D_{63}^{2} t(11111111) .
$$

(Notice here $e_{0}=2, e_{1}=0, e_{2}=3$, etc., and that this correspondence is best conceived using reverse binary digit representations.)

An analogous phenomenon in the context of finite $H$-spaces was already observed and illustrated in [5, p. 1490f].

In general we now clearly have

Theorem 2.1 (A basis for $H^{*} R P(\infty)$ in terms of $\mathcal{K}$ ). For $j \geqslant 0$, a basis for

$$
F_{j} H^{*} \mathbb{R} P(\infty) / F_{j-1} H^{*} \mathbb{R} P(\infty)
$$

in terms of admissibles from $\mathcal{K}$ on $t^{2^{j}-1}$ is given by

$$
\left\{D_{0}^{e_{0}} \cdots D_{2^{i}-1}^{e_{i}} \cdots D_{2^{j-1}-1}^{e_{j-1}} t^{2^{j}-1} \mid \text { each } e_{i} \geqslant 0\right\},
$$

with corresponding degrees $\sum_{i=0}^{j-1} 2^{e_{0}+\cdots+e_{i}+i}$ as in displayed equation (2) above. 
This form of basis thus allows us to state specific module relations between the minimal generators $\left\{t^{2^{j}-1} \mid j \geqslant 0\right\}$ in $H^{*} \mathbb{R} P(\infty)$, based on the preliminary calculations above of candidates for a minimal set of module relations:

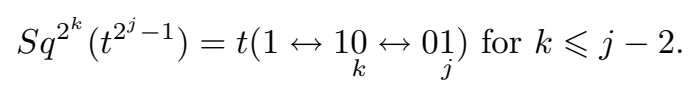

Thus we have

$$
S q^{2^{k}} t^{2^{j}-1}=D_{2^{k}-1}^{j-k} t^{2^{k+1}-1} \text { for } k \leqslant j-2
$$

\section{Minimal module presentations for the filtrations of $H^{*} \mathbb{R} P(\infty)$}

From directly above, our theorem about $F_{s} H^{*} \mathbb{R} P(\infty)$ will be

Theorem 3.1 (Minimal presentation for $F_{s} H^{*} R P(\infty)$ ). For $0 \leqslant s \leqslant \infty$, a minimal presentation of $F_{s} H^{*} \mathbb{R} P(\infty)$ as an unstable module is given by generators

$x_{2^{j}-1}, \quad$ for $j$ an integer with $0 \leqslant j \leqslant s \quad$ (degree of $x_{2^{j}-1}$ is $2^{j}-1$ ),

and relations

$$
S q^{2^{k}} x_{2^{j}-1}=D_{2^{k}-1}^{j-k} x_{2^{k+1}-1}, \text { for } 0 \leqslant j \leqslant s \text { and } 0 \leqslant k \leqslant j-2 .
$$

Remark. Already in the introduction we noted that these relations can be recast solely in terms of the $\mathcal{A}$-action. They are equal to the set

$$
S q^{2^{k}} x_{2^{j}-1}=S q^{2^{j-1}} S q^{2^{j-2}} \cdots S q^{2^{k}} x_{2^{k+1}-1} \text { for } 0 \leqslant j \leqslant s \text { and } 0 \leqslant k \leqslant j-2,
$$

and equivalent to the set

$$
S q^{2^{k}} x_{2^{j}-1}=S q^{2^{j-1}} S q^{2^{k}} x_{2^{j-1}-1} \text { for } 0 \leqslant j \leqslant s \text { and } 0 \leqslant k \leqslant j-2 .
$$

In preparation for the proof of Theorem 3.1, notice that from above we already have a basis in terms of $\mathcal{K}$ for the filtration quotients $F_{j} / F_{j-1}$ of $H^{*} \mathbb{R} P(\infty)$. Therefore, since our goal is to present $F_{s} H^{*} \mathbb{R} P(\infty)$ via generators and relations, we next endeavor to identify abstract minimal $\mathcal{K}$-presentations for these filtration quotients $F_{j} / F_{j-1}$. These abstract presentations, when recast via $\mathcal{A}$-presentations, will perfectly match the obvious filtered quotients of the presentation in the theorem, and the rest will follow easily.

\subsection{A third point of view: analyze as a $\mathcal{K}$-module}

We wish to prove (cf. [6, Theorems 6.1, 6.2]).

Theorem 3.2 (Filtration quotients presented as cyclic modules). For $j \geqslant 0$, the filtered quotient $F_{j} H^{*} \mathbb{R} P(\infty) / F_{j-1} H^{*} \mathbb{R} P(\infty)$ is $\mathcal{A}$-isomorphic to the free unstable cyclic module on a generator $x_{2^{j}-1}$ in degree $2^{j}-1$, modulo the left ideal generated by $\left\{S q^{2^{k}} \mid k \leqslant j-2\right\}$, which module we denote by $\mathcal{M}(j, 1)$ for consistency with [6]. The abstract cyclic module $\mathcal{M}(j, 1)$ thus has basis as described in Theorem 2.1, with $x_{2^{j}-1}$ replacing $t^{2^{j}-1}$. In particular, the abstract cyclic module is nonzero, and of rank one, precisely in those degrees with $\alpha$-number $j$. 
Our method of proof will go beyond just describing elements in these $\mathcal{A}$-modules via $\mathcal{K}$-operations and a basis in terms of $\mathcal{K}$. We will actually shift to viewing these unstable $\mathcal{A}$-modules equivalently as $\mathcal{K}$-modules, since there is a special feature of the product structure of $\mathcal{K}$ that we wish to exploit. The existence of this feature, inherent to the algebraic structure of $\mathcal{K}$ as opposed to that of $\mathcal{A}$, demonstrates why shifting to the $\mathcal{K}$-module view is important.

Notation. Let $\mathcal{F}_{x_{2^{j}-1}}$ denote the free unstable module on $x_{2^{j}-1}$ in degree $2^{j}-1$.

To prove Theorem 3.2, indeed to obtain a basis for

$$
\mathcal{F}_{x_{2^{j}-1}} / \mathcal{A}\left(S q^{2^{k}} \mid k \leqslant j-2\right) x_{2^{j}-1}
$$

written in terms of $\mathcal{K}$, we will analyze it by shifting to view it as a $\mathcal{K}$-module, for which purpose we make two definitions.

Definition. Let $\mathcal{I}_{j}=\mathcal{A}\left(S q^{2^{k}} \mid k \leqslant j-2\right)$, a left ideal in $\mathcal{A}$ depending on $j$.

Definition. Let $\mathcal{J}=\mathcal{K}\left(D_{i} \mid i\right.$ is not of the form $2^{l}-1$ for any $\left.l\right)$, a left ideal in $\mathcal{K}$ not depending on $j$.

Despite the fact that $\mathcal{J}$ does not depend on $j$, while $\mathcal{I}_{j}$ does, we can prove that

Theorem 3.3 (Shift presentation to $\mathcal{K}$-module). For each $j \geqslant 0$, in $\mathcal{F}_{x_{2^{j}-1}}$ the submodules $\mathcal{I}_{j} x_{2^{j}-1}$ and $\mathcal{J} x_{2^{j}-1}$ are equal. Equivalently,

$$
\mathcal{F}_{x_{2^{j}-1}} / \mathcal{A}\left(S q^{2^{k}} \mid k \leqslant j-2\right) x_{2^{j}-1}=\mathcal{F}_{x_{2^{j}-1}} / \mathcal{K}\left(D_{i} \mid i \neq 2^{l}-1\right) x_{2^{j}-1} .
$$

This converts the minimal $\mathcal{A}$-presentation of this abstract module into a minimal $\mathcal{K}$-presentation.

Remark. To provide some motivation for why these very different looking quotients might be the same, consider which Steenrod squares we expect to survive on the left side. Certainly $S q^{2^{j-1}} x_{2^{j}-1}$ survives, and we note it equals $D_{2^{j-1}-1} x_{2^{j}-1}$, which also survives on the right. Those familiar with the Adem relations in $\mathcal{A}$ will also expect that $S q^{2^{j-1}+2^{j-2}} x_{2^{j}-1}$ may survive on the left, and it equals the survivor $D_{2^{j-2}-1} x_{2^{j}-1}$ on the right. This pattern continues.

Proof. We show bicontainment for the generators of the two left ideals.

For $k \leqslant j-2$, we consider the generator $S q^{2^{k}}$ of $\mathcal{I}_{j}$. Notice that $S q^{2^{k}} x_{2^{j}-1}=$ $D_{2^{j}-2^{k}-1} x_{2^{j}-1}$, and $2^{j}-2^{k}-1$ is not of the form $2^{l}-1$, so $D_{2^{j}-2^{k}-1}$ is a generator of $\mathcal{J}$.

In the other direction, let $r<2^{j}-1$ be not of the form $2^{l}-1$ for any $l$, and consider $D_{r} x_{2^{j}-1}$ in $\mathcal{F}_{x_{2^{j}-1}} / \mathcal{A}\left(S q^{2^{k}} \mid k \leqslant j-2\right) x_{2^{j}-1}=\mathcal{M}(j, 1)$. To show that $D_{r} x_{2^{j}-1}=0 \in$ $\mathcal{M}(j, 1)$, we will induct downwards on $r$. If $2^{j-1}-1<r<2^{j}-1$, then $D_{r} x_{2^{j}-1}=$ $S q^{m} x_{2^{j}-1}$ with $0<m<2^{j-1}$, which is zero from the defining relations of $\mathcal{M}(j, 1)$. Continuing downwards, suppose that $r<2^{j-1}-1$. Then we can write $r=\left(2^{j}-1\right)-$ $\left(2^{a} c+2^{b}\right)$, with $c$ odd and $0 \leqslant b<a \leqslant j-1$. We consider two cases:

Case 1. Let $\left(2^{j}-1\right)-2^{a} c \neq 2^{l}-1$, for any $l$. A calculation with the $\mathcal{K}$ Adem relations, combined with the unstable condition and the inductive hypothesis, yields

$$
D_{r+2^{a+1}{ }_{c}} D_{\left(2^{j}-1\right)-2^{a} c} x_{2^{j}-1}=D_{r} D_{2^{j}-1} x_{2^{j}-1}=D_{r} x_{2^{j}-1} .
$$

Since $\left(2^{j}-1\right)-2^{a} c>r$, inductively we have $D_{r} x_{2^{j}-1}=0$. 
Case 2. Let $\left(2^{j}-1\right)-2^{a} c=2^{l}-1$ for some $l$. It follows that $l=a$ and that $c=$ $2^{j-a}-1$. Again using the Adem relations in $\mathcal{K}$, combined with the unstable condition, the inductive hypothesis, and the assumption that $r$ is not of the form $2^{l}-1$ for any $l$, we get

$$
0=D_{2^{j+1}-2^{a}-3 \cdot 2^{b}-1} D_{2^{a}+2^{b}-1} x_{2^{j}-1}=D_{r} D_{2^{j}-1} x_{2^{j}-1}=D_{r} x_{2^{j}-1} .
$$

Now we recall a remarkable feature of the left ideal $\mathcal{J}$ in $\mathcal{K}$, for which we know no analog in $\mathcal{A}$.

Theorem 3.4 (Quasi-two-sided ideal in $\mathcal{K}$; no apparent $\mathcal{A}$ analog). The left ideal $\mathcal{J}$ is quasi-two-sided in $\mathcal{K}$, i.e.,

$$
\left\{\text { admissible } D_{i_{1}} \cdots D_{i_{r}} \cdots D_{i_{q}} \mid \text { at least one } D_{i_{r}} \text { is in } \mathcal{J}\left(i . e ., i_{r} \neq 2^{l}-1\right)\right\}
$$

is contained in $\mathcal{J}$.

In other words, any admissible in $\mathcal{K}$ with a generator of $\mathcal{J}$ anywhere in its product can be written in terms of elements with generators of $\mathcal{J}$ on the right.

This property of $\mathcal{K}$ was proven from the $\mathcal{K}$-Adem relations in Theorem 2.9 of [7].

The preceding two theorems now come together with Theorem 2.1 to prove Theorem 3.2 .

Proof of Theorem 3.2. First note that $\left\{S q^{2^{k}} \mid k \leqslant j-2\right\}$ acts trivially on $t^{2^{j}-1} \in$ $F_{j} / F_{j-1}$, since $F_{j} / F_{j-1}$ is zero in the target degrees. Thus there is a (unique) $\mathcal{A}$ map $\psi_{j}: \mathcal{M}(j, 1) \rightarrow F_{j} / F_{j-1}$ with $\psi_{j}\left(x_{2^{j}-1}\right)=t^{2^{j}-1}$. From Theorems 2.1 and 3.3 , we have a module epimorphism

$$
\begin{aligned}
\mathcal{F}_{x_{2^{j}-1}} / \mathcal{K}\left(D_{i} \mid i \neq 2^{l}-1\right) x_{2^{j}-1} & =\mathcal{F}_{x_{2^{j}-1}} / \mathcal{A}\left(S q^{2^{k}} \mid k \leqslant j-2\right) x_{2^{j}-1} \\
& =\mathcal{M}(j, 1) \\
& \stackrel{\psi_{j}}{\rightarrow} F_{j} / F_{j-1} \\
& =\mathbb{F}_{2}\left\{D_{0}^{e_{0}} \cdots D_{2^{i}-1}^{e_{i}} \cdots D_{2^{j-1}-1}^{e_{j-1}} t^{2^{j}-1} \mid e_{i} \geqslant 0\right\} .
\end{aligned}
$$

Moreover, from Theorem 3.4, every admissible in $\mathcal{K}$ (assumed ending in $D_{r}$ with $r<2^{j}-1$ for unstable nontriviality and nonredundancy on a class in degree $2^{j}-1$ ), other than those appearing in the basis for $F_{j} / F_{j-1}$, actually produces zero on $x_{2^{j}-1}$. So the map is an isomorphism.

From the proof of the theorem we also have

Corollary. For $j \geqslant 0$, all admissibles in $\mathcal{K}$ (assumed ending in $D_{r}$ with $r<2^{j}-1$ for unstable nontriviality and nonredundancy on a class in degree $2^{j}-1$ ), other than those shown in the basis above, are zero on both the fundamental class $t^{2^{j}-1}$ in the cyclic module $F_{j} H^{*} \mathbb{R} P(\infty) / F_{j-1} H^{*} \mathbb{R} P(\infty)$ and the fundamental class $x_{2^{j}-1}$ in the abstract cyclic module $\mathcal{M}(j, 1)$.

We end this section by proving the minimal presentations for $F_{s} H^{*} \mathbb{R} P(\infty)$.

Proof of Theorem 3.1. First we create notation for the claimed module presentation, and observe that desired maps exist between the presentation and the filtrations in $H^{*} \mathbb{R} P(\infty)$. 
Let $\mathcal{N}_{s}(s \geqslant 0)$ denote the free unstable module on the set

$$
x_{2^{j}-1} \text {, for } 0 \leqslant j \leqslant s \quad\left(\text { degree of } x_{2^{j}-1} \text { is } 2^{j}-1\right),
$$

subject to the relations

$$
S q^{2^{k}} x_{2^{j}-1}=D_{2^{k}-1}^{j-k} x_{2^{k+1}-1}, \text { for } 0 \leqslant j \leqslant s, 0 \leqslant k \leqslant j-2 .
$$

We verified earlier that the images of these relations are satisfied in $F_{s}$, so there are epimorphisms of unstable modules $\varphi_{s}: \mathcal{N}_{s} \rightarrow F_{s}$ with $\varphi_{s}\left(x_{2^{j}-1}\right)=t^{2^{j}-1}$ for all $0 \leqslant j \leqslant s$, such that the diagram

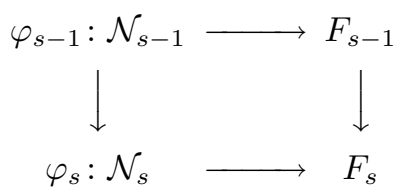

commutes, where $\mathcal{N}_{s-1} \rightarrow \mathcal{N}_{s}$ is the obvious map between these presentations.

Inductively assume that $\varphi_{s-1}$ is an isomorphism. It follows from the diagram that $\mathcal{N}_{s-1} \rightarrow \mathcal{N}_{s}$ is monic. We shall prove that $\varphi_{s}$ is an isomorphism by showing that the induced map $\mathcal{N}_{s} / \mathcal{N}_{s-1} \rightarrow F_{s} / F_{s-1}$ is an isomorphism. Clearly an unstable presentation for $\mathcal{N}_{s} / \mathcal{N}_{s-1}$ consists of the single generator $x_{2^{s-1}}$ and relations $S q^{2^{k}} x_{2^{s-1}}=0$, for $0 \leqslant k \leqslant s-2$, yielding $\mathcal{M}(s, 1)$. So the induced map is an isomorphism by Theorem 3.2.

Minimality of the set of relations is clear from looking at filtration quotients, since the $S q^{2^{k}}$ minimally generate $\mathcal{A}$.

\section{Minimal module presentations of $H^{*} \mathbb{R} P(m)$}

With Theorem 3.1 in hand we can turn to minimal $\mathcal{A}$-module presentations for the finite projective spaces. We illustrate first with a special case, presenting $H^{*} \mathbb{R} P(m)$ for the particular values $m$ of the form $2^{s+1}-2$.

Proposition 4.1 (Presentation for a special family of finite projective spaces). $A$ minimal presentation of $H^{*} \mathbb{R} P\left(2^{s+1}-2\right)$ as an unstable $\mathcal{A}$-module is given by generators

$$
x_{2^{j}-1} \text { in degree } 2^{j}-1 \text {, for } 0 \leqslant j \leqslant s \text {, }
$$

and relations

$$
S q^{2^{k}} x_{2^{j}-1}=D_{2^{k}-1}^{j-k} x_{2^{k+1}-1}, \text { for } 0 \leqslant j \leqslant s \text { and } 0 \leqslant k \leqslant j-2
$$

and

$$
0=D_{2^{k}-1}^{s+1-k} x_{2^{k+1}-1}, \text { for } 0 \leqslant k \leqslant s-1 .
$$

Proof. Clearly $H^{*} \mathbb{R} P\left(2^{s+1}-2\right)$ is the quotient of $F_{s}=F_{s} H^{*} \mathbb{R} P(\infty)$ obtained by killing all $t^{r}$ in $F_{s}$ with $r \geqslant 2^{s+1}-1$. So the generators and relations from Theorem 3.1 for $F_{s}$ must all necessarily appear, since they lie in degrees lower than $2^{s+1}-1$. It is not immediately clear what minimal set of $\mathcal{A}$-relations should then additionally be imposed in $F_{s}$ to achieve triviality in degrees above $2^{s+1}-1$ (in degree $2^{s+1}-1$, 
$F_{s}$ is zero). We consider the least such degrees nonzero in $F_{s}$ with given numbers of trailing ones, namely

$$
\begin{aligned}
& (0 \leftrightarrow 0 \underset{s+1}{1}) \\
& \text {... } \\
& (1 \leftrightarrow \underset{k}{10} \leftrightarrow 0 \underset{s+1}{0}) \\
& \text {... } \\
& \left(\begin{array}{lllll}
1 \leftrightarrow & 4 & 0 & 0 & 1 \\
s-1 & s+1
\end{array}\right) .
\end{aligned}
$$

It is clear that each of these degrees holds a necessary relation, being the lowest degree above $2^{s+1}-1$ with its number of trailing ones, and thus unreachable from any others by shoveling. But clearly also any element of $F_{s}$ in any degree above $2^{s+1}-1$ can be reached from one of these by shoveling, i.e. (using Theorem 2.1), from the set of elements of the form $D_{2^{k}-1}^{s+1-k} x_{2^{k+1}-1}$ in $F_{s}$. This is precisely the second set of relations.

A minimal presentation of $H^{*} \mathbb{R} P(m)$ for arbitrary $m$ is more complicated. It will need new relations accounting for the deviation of $m$ below the nearest higher number of the form $2^{s+1}-2$, but will still utilize a subset of the second set of relations used above to present $H^{*} \mathbb{R} P\left(2^{s+1}-2\right)$.

Theorem 4.2 (Minimal presentation for finite projective spaces). Let $2^{s}-1 \leqslant m \leqslant$ $2^{s+1}-2$. Let $\alpha$ be the number of ones in the binary expansion of $m+1$, and write the expansion as $m+1=\sum_{i=0}^{\alpha-1} 2^{e_{0}+\cdots+e_{i}+i}$ (each $e_{i} \geqslant 0$ ). Also let $\beta$ be the number of leading ones in this expansion; in other words, if there is a nonzero $e_{i}$, then $\alpha-\beta$ is the largest index $i$ for which $e_{i}$ is nonzero.

Then a minimal presentation for $H^{*} \mathbb{R} P(m)$ is given by generators

$$
x_{2^{j}-1} \text { in degree } 2^{j}-1 \text {, for } 0 \leqslant j \leqslant s,
$$

and the three sets of relations

$$
\begin{gathered}
S q^{2^{k}} x_{2^{j}-1}=D_{2^{k}-1}^{j-k} x_{2^{k+1}-1}, \text { for } 0 \leqslant j \leqslant s \text { and } 0 \leqslant k \leqslant j-2, \\
0=D_{2^{i+r}-1}^{e_{i}-l} D_{2^{i+1+r}-1}^{e_{i+1}} \cdots D_{2^{\alpha-1+r}-1}^{e_{\alpha-1}} x_{2^{\alpha+r}-1}, \\
\text { for } 0 \leqslant i \leqslant \alpha-1 \text { and } 0 \leqslant l<e_{i} \text {, where } r=e_{0}+\cdots+e_{i-1}+l \text {, and } \\
0=D_{2^{k}-1}^{s+1-k} x_{2^{k+1}-1}, \text { for } s+1-\beta \leqslant k \leqslant s-1 .
\end{gathered}
$$

Remark. These relations may be demystified by observing, as will be explained in the proof, that the second set of relations consists of elements in degrees obtained by beginning with degree $m+1$, then successively replacing zeros in its binary expansion by ones, starting from the left, until only one zero remains, so as to stay inside $F_{s}$. And the third set then continues above the next power of two in all the lowest degrees with more trailing ones than the second set, but stopping with two zeros, again to stay inside $F_{s}$. The proof spells this out in detail. 
Example. If $m+1=(1010011100111)$, the second set of relations lies in the degrees (1110011100111), (1111011100111), (1111111100111), and (1111111110111), and the third set adds more trailing ones in degrees (11111111110001) and (11111111111001) above the next power of two.

Remark. The first and third sets of relations arise from the status of $H^{*} \mathbb{R} P(m)$ as the quotient of $H^{*} \mathbb{R} P\left(2^{s+1}-2\right)$, which latter was minimally presented in Proposition 4.1. The second set of relations in the general theorem truncates the cohomology down further from $H^{*} \mathbb{R} P\left(2^{s+1}-2\right)$ to $H^{*} \mathbb{R} P(m)$, and the minimal third set of relations is only a subset of the second set in Proposition 4.1, since the addition of the second set of relations for general $m$ makes some of the second set of relations for $H^{*} \mathbb{R} P\left(2^{s+1}-2\right)$ from Proposition 4.1 redundant for minimally presenting $H^{*} \mathbb{R} P(m)$, based on how many trailing ones can appear in the second set for $H^{*} \mathbb{R} P(m)$.

The extreme case $m=2^{s+1}-2$ corresponds precisely to $\beta=s+1$, in which case all the $e_{i}$ are zero, there are no relations in the second set, and the third set is the full set from Proposition 4.1. At the other extreme, if $\beta=1$, then the third set is empty; this corresponds to the first half of each degree range for $m$.

Proof of Theorem 4.2. Initially we impose the first set of relations in the theorem. From Theorem 3.1, the resulting quotient provides a minimal presentation for the filtration $F_{s}$ of $H^{*} \mathbb{R} P(\infty)$. Hence we may now use the formulas for the unstable action in $H^{*} \mathbb{R} P(\infty)$, in particular we may shovel ones. And furthermore, in degrees less than $2^{s+1}$, this initial quotient, isomorphic to $F_{s}$, is also isomorphic to $H^{*} \mathbb{R} P\left(2^{s+1}-2\right)$.

The remaining two sets of relations are to minimally ensure that the final result is zero in all degrees greater than or equal to $m+1$. Note that the third set of relations in the theorem lies in degrees at least $2^{s+1}$, as seen in the proof of Proposition 4.1. Thus we proceed first with relations in the range $2^{s}-1 \leqslant m \leqslant 2^{s+1}-2$ within which $m$ lies, and in which range the presentation so far agrees with both $F_{s}$ and $H^{*} \mathbb{R} P\left(2^{s+1}-2\right)$, having a single element in each degree.

Consider the expansion

$$
m+1=\sum_{i=0}^{\alpha-1} 2^{e_{0}+\cdots+e_{i}+i}=(\underbrace{0 \leftrightarrow 0}_{e_{0}} 1 \cdots 1 \underbrace{0 \leftrightarrow 0}_{e_{i}} 1 \cdots 1 \underbrace{0 \leftrightarrow 0}_{e_{\alpha-1}} 1),
$$

with $\alpha$-many ones. We need only identify sequentially by degree which elements need to be killed in this range to produce $H^{*} \mathbb{R} P(m)$, simultaneously tracking which elements will be zero in the developing quotient as we inductively impose relations in lower degrees. This will all be determined by how the $\mathcal{A}$-action, generated by shoveling, does or does not connect one degree to another in $F_{s}$. Then we can write the explicit relations to kill these in terms of our canonical basis in terms of $\mathcal{K}$ for all elements of $F_{s}$.

Note first that if $m=2^{s+1}-2$, then $m+1=2^{s+1}-1$ is already outside our range, so no relations in this range will be needed. However, if $m<2^{s+1}-2$, then after killing the element in degree $m+1$ itself, it is clear that the list of additional necessary and sufficient relations in this range must be in those degrees obtained from the binary expansion of $m+1$ by successively replacing its zeros by ones, from left to right. This is because any given element in the range is clearly in the $\mathcal{A}$-image of 
the element in this list with degree closest below the given element. Moreover, none of the elements of increasing degree in this list can be reached from each other by shoveling, since their degrees' reversed binary expansions begin with ever increasing numbers of trailing ones. Finally, note that this list stops when there is a single zero remaining, namely the rightmost zero in the original expansion. Otherwise the degree would become $2^{s+1}-1$, outside the range. Using the correspondence of Theorem 2.1 between degrees and a basis in terms of $\mathcal{K}$ for $H^{*} \mathbb{R} P(\infty)$, these relations are precisely those in the second set in the theorem. Note that in the degree of each relation, $r$ represents the number of zeros in the expansion of the degree $m+1$ being replaced by ones. Note too that some of the $e_{i}$ may be zero, reflecting consecutive ones in the expansion of $m+1$, producing corresponding empty factors in the basis descriptions in terms of $\mathcal{K}$, except for the leading factor, for which the exponent $e_{i}-l$ is always positive.

Finally we consider what relations are still needed above degree $2^{s+1}-1$ in order to ensure that the quotient is zero there. Clearly this will be some subset of the set of relations added in this range for $H^{*} \mathbb{R} P\left(2^{s+1}-2\right)$ in Proposition 4.1. The final relation we just added in the second set of relations has the largest number of trailing ones in its binary expansion. Recalling the definition of $\beta$ in the theorem, this final relation in the second set is in degree

$$
\left.(1 \leftrightarrow 1 \underset{s-\beta}{0} 1 \leftrightarrow)_{s}\right)
$$

Clearly any element of $F_{s}$ in higher degree than this, and with no more trailing ones, can be reached by shoveling from the element in this degree. So it remains only to deal with elements in degrees with more trailing ones, for which we consider the elements in degrees

$$
\begin{aligned}
& (1 \leftrightarrow 1 \underset{s-\beta+1}{0} \leftrightarrow 0 \underset{s+1}{0}) \\
& \text {... } \\
& \left(1 \leftrightarrow \underset{k}{10} \leftrightarrow 0_{s+1}^{1}\right) \\
& \text {... } \\
& \left(\begin{array}{llll}
1 \leftrightarrow & 0 & 0 & 1 \\
s-1 & & 1
\end{array}\right)
\end{aligned}
$$

in $F_{s}$. These are precisely the lowest degrees above $2^{s+1}-1$ in $F_{s}$ with more trailing ones, so they are all necessary relations. (Note that this is also correct for the case when there are no type two relations, i.e., when $m=2^{s+1}-2, \beta=s+1$, and the relations above include all possible numbers of trailing ones, as necessary.) But they are also sufficient, since clearly any element of $F_{s}$ in any degree above $2^{s+1}-1$ with as many trailing ones as one of these can be reached from one of these by shoveling. So again using the correspondence of Theorem 2.1, we set the elements in these degrees to zero to write the third set of relations in the theorem.

\section{References}

[1] H.E.A. Campbell, F.R. Cohen, F.P. Peterson and P.S. Selick, Self-maps of loops spaces. II, Trans. Amer. Math. Soc. 293 (1986), no. 1, 41-51. 
[2] H.E.A. Campbell, F.P. Peterson and P.S. Selick, Self-maps of loops spaces. I, Trans. Amer. Math. Soc. 293 (1986), no. 1, 1-39.

[3] F.R. Cohen, T.J. Lada and J.P. May, The homology of iterated loop spaces, Lecture Notes in Mathematics 533, Springer-Verlag, New York (1976).

[4] I. Madsen and R.J. Milgram, The classifying spaces for surgery and cobordism of manifolds, Annals of Mathematics Studies 92, Princeton Univ. Press, Princeton, NJ, 1979.

[5] D.J. Pengelley and F. Williams, Sheared algebra maps and operation bialgebras for mod 2 homology and cohomology, Trans. Amer. Math. Soc. 352 (2000), no. $4,1453-1492$.

[6] D.J. Pengelley and F. Williams, Global structure of the mod two symmetric algebra, $H^{*}\left(B O ; \mathbb{F}_{2}\right)$, over the Steenrod Algebra,Algebraic \& Geometric Topology 3 (2003), 1119-1138.

[7] D.J. Pengelley, F.P. Peterson and F. Williams, A global structure theorem for the mod 2 Dickson algebras, and unstable cyclic modules over the Steenrod and Kudo-Araki-May algebras, Math. Proc. Cambridge Philos. Soc. 129 (2000), no. 2, 263-275.

[8] N.E. Steenrod and D.B.A. Epstein, Cohomology operations, Annals of Mathematics Studies 50, Princeton Univ. Press, Princeton, NJ, 1962.

David J. Pengelley davidp@nmsu.edu

Department of Mathematical Sciences, New Mexico State University, Las Cruces, NM 88003, USA

Frank Williams frank@nmsu.edu

Department of Mathematical Sciences, New Mexico State University, Las Cruces, NM 88003, USA 